\title{
Compression-induced texture change in NiMnGa-polymer composites observed by synchrotron radiation
}

\author{
Nils Scheerbaum, ${ }^{\text {a) }}$ Dietrich Hinz, and Oliver Gutfleisch \\ Institute for Metallic Materials, IFW Dresden, P.O. Box 270116, D-01171 Dresden, Germany \\ Werner Skrotzki \\ Institut für Strukturphysik, Technische Universität Dresden, D-01062 Dresden, Germany \\ Ludwig Schultz \\ Institute for Metallic Materials, IFW Dresden, P.O. Box 270116, D-01171 Dresden, Germany
}

(Presented on 10 January 2007; received 31 October 2006; accepted 8 November 2006; published online 22 March 2007)

Composites consisting of magnetic shape memory (MSM) particles embedded in a polyester matrix were prepared. Single-crystalline MSM particles were obtained by mortar grinding of melt-extracted and subsequently annealed $\mathrm{Ni}_{50.9} \mathrm{Mn}_{27.1} \mathrm{Ga}_{22.0}$ (at. \%) fibers. The crystal structure of the martensite is tetragonal $(5 M)$ with $c<a=b$. Magnetic characterization of these composites shows indirect evidence for stress induced twin boundary motion in the MSM particles, as the compressed composite is easy to magnetize in the direction of compression and more difficult to magnetize in the perpendicular directions. The texture of all the embedded MSM particles is investigated before and after compression by means of synchrotron radiation. In the initial state, the MSM particles in the composite have a random texture, i.e., there is no preferred orientation of the $c$ axis. After a 30\% compression (height reduction), the MSM particles have a (004)-fiber texture in the direction of compression. This is unambiguous evidence for stress induced twin boundary motion within the MSM particles. (C) 2007 American Institute of Physics. [DOI: 10.1063/1.2670414]

The term magnetic shape memory (MSM) effect refers to a magnetic field induced structural phase transformation. At the same time, also a magnetic field induced twin boundary motion is commonly referred to as MSM effect. ${ }^{1-3}$ Large magnetic field induced strains can be achieved in MSM alloys, e.g., in the Heusler alloy $\mathrm{Ni}_{2} \mathrm{MnGa}$ up to $10 \%{ }^{4}$ Besides employing single and polycrystals to utilize the MSM effect in actuators or dampers, also composites, i.e., MSM particles embedded in a polymer matrix, were proposed. ${ }^{5,6}$ It was shown previously that compressed composite samples can be magnetized easier in the compressed direction and it is more difficult to magnetize them in the perpendicular directions. ${ }^{6,7}$ This behavior was regarded as an indication for stress induced twin boundary motion within the MSM particles.

In order to verify this, the texture of the MSM particles before and after compression is investigated in this work. The texture is measured by using diffraction of high-energy synchrotron radiation. The advantage of this method is that the texture of the whole sample volume is measured.

The MSM particles used were prepared by mortar grinding of crucible melt-extracted and subsequently annealed $\mathrm{Ni}_{50.9} \mathrm{Mn}_{27.1} \mathrm{Ga}_{22.0}$ (at. \%) fibers. The preparation of the composite was done by mixing these MSM particles with a two component polyester resin (about $20 \mathrm{vol} \%$ MSM particles) followed by a vacuum treatment to remove air bubbles. Curing of the polyester matrix was done without a magnetic field applied. The composite was disk shaped with a height of $2.79 \mathrm{~mm}$ and a diameter of about $9 \mathrm{~mm}$. A cylindrical

\footnotetext{
${ }^{a)}$ Author to whom correspondence should be addressed; electronic mail: n.scheerbaum@ifw-dresden.de
}

sample with a diameter of $3.03 \mathrm{~mm}$ and a height of $2.79 \mathrm{~mm}$ was cut out from the middle of the composite. Detailed information on preparation of MSM particles and composites are given by Scheerbaum et al. ${ }^{6}$ For the synchrotron texture measurements the beam line BW5 (about $100 \mathrm{keV}$ ) at DESY-HASYLAB in Hamburg (Germany) was used. The synchrotron radiation is diffracted on the lattice planes of all MSM particles. The diffraction cones with an aperture angle of $4 \Theta$ are imaged on an area detector mounted behind the sample. The whole texture information is collected by rotating the sample in $3^{\circ}$ steps from $0^{\circ}$ to $180^{\circ}$ and recording after each step the image on the area detector. Details about the method are given by Skrotzki et al. ${ }^{8,9}$ The texture was measured before compression on the cylindrical composite sample mentioned above (diameter $3.03 \mathrm{~mm}$, height $2.79 \mathrm{~mm}$. Afterwards the sample was compressed along the cylinder axis $Z$ by about $30 \%$. After removing the stress about $2 / 3$ of the strain relaxed leaving a permanent compression of about $10 \%$. The sample size for the following texture measurement was $3.20 \mathrm{~mm}$ in diameter and $2.53 \mathrm{~mm}$ in height.

The magnetic field $H$ dependent magnetization $M$ of the composites was measured before and after compression parallel and perpendicular to the compression direction. For these $M-H$ measurements a vibrating sample magnetometer (VSM) was used. Here only the demagnetization curves are shown, but the magnetization curves exhibit, apart from a slight shift, the same behavior.

After annealing and mortar grinding, the MSM particles are about $50-100 \mu \mathrm{m}$ in size and are either single crystalline (fraction about $20 \%-40 \%$ ) or are composed of a few grains. 

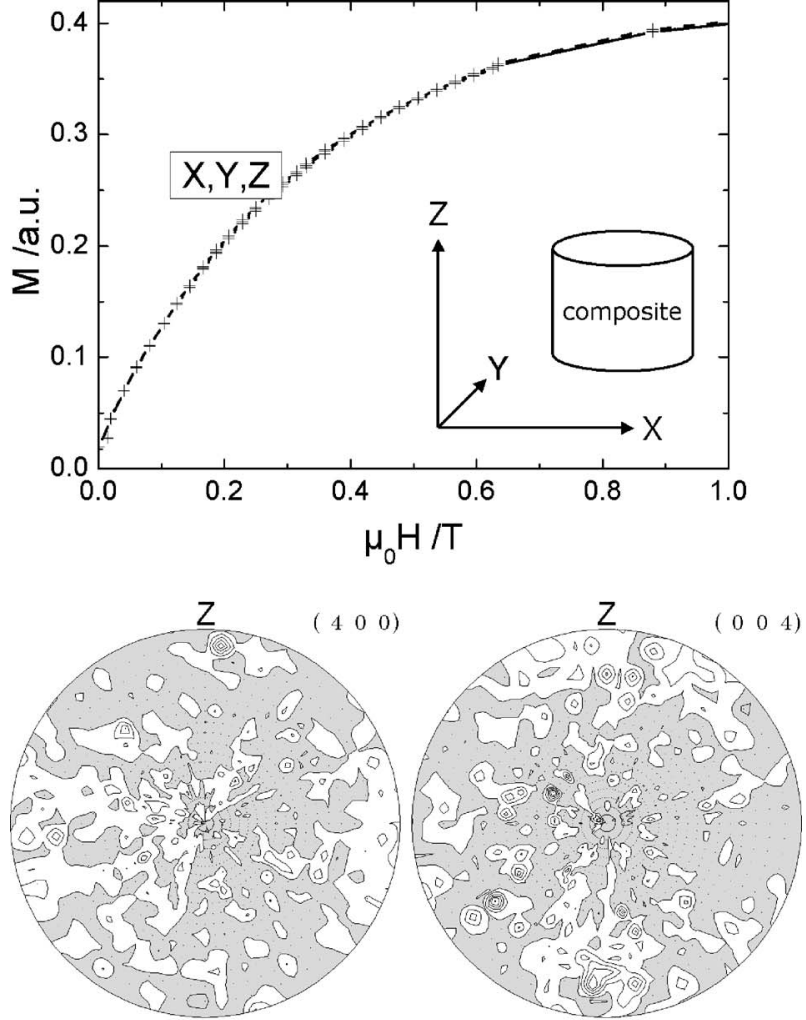

$\begin{array}{lllllllllllll}0.50 & 1.00 & 1.50 & 2.00 & 2.50 & 3.00 & 0.50 & 1.00 & 1.50 & 2.00 & 2.50 & 3.00\end{array}$ $\min =0.27$ $\max =3.53 \stackrel{4.00}{\min =0.31}$

$\max =4.17$

FIG. 1. Composite sample before compression. The $M-H$ curves (top) are the same for all three directions. The (400) and the (004) pole figures (bottom) correspond to the $a / b$ axes and to the $c$ axis, respectively. There is no preferred orientation visible.

Their martensitic transformation and Curie temperatures are well above room temperature $\left(M_{s}=45^{\circ} \mathrm{C}, M_{f}=37^{\circ} \mathrm{C}, A_{s}\right.$ $=54{ }^{\circ} \mathrm{C}, A_{f}=61{ }^{\circ} \mathrm{C}$, and $T_{C}=98{ }^{\circ} \mathrm{C}$, respectively), and the martensite has the tetragonal crystal structure $(c<a \cong b)$ with the modulation type $5 M$ (unit cell describing $5 M$ modulation: $a^{\prime}=0.422 \mathrm{~nm}, b^{\prime}=5 \times 0.4194 \mathrm{~nm}=2.097 \mathrm{~nm}, c^{\prime}=c$ $=0.558 \mathrm{~nm}$, and $\gamma^{\prime}=90.23^{\circ} ; \alpha^{\prime}, \beta^{\prime}$ nearly $\left.90^{\circ}\right)$. Detailed in formation on the properties of the MSM particles can be found in Ref. 6.

Figure 1 shows the $M-H$ curves (top) and the pole figures (bottom) of the composite sample before compression. All three directions have identical magnetization behavior and no preferred orientation is visible in the pole figures. Thus, the crystallographic orientation of the MSM particles within the composite, and therefore of the easy magnetization axis $c$, is random. This changes after compression along $Z$ (Fig. 2). As previously shown, ${ }^{6}$ the composite sample is now easier to magnetize in $Z$ direction than in the other two perpendicular directions. The higher the deformation, the easier becomes the $Z$ direction to magnetize. ${ }^{7}$ The pole figures show a (004)-fiber texture along the $Z$ direction, verifying that the $c$ axis actually aligns with the compression direction $Z$. The $c$ axis can either be reoriented by rotation or by twin boundary motion within the MSM particles. Rotation can be excluded as a mere rotation of the MSM particles within the matrix due to compression would not lead to a preferred orientation of the $c$ axes (assuming a sufficient
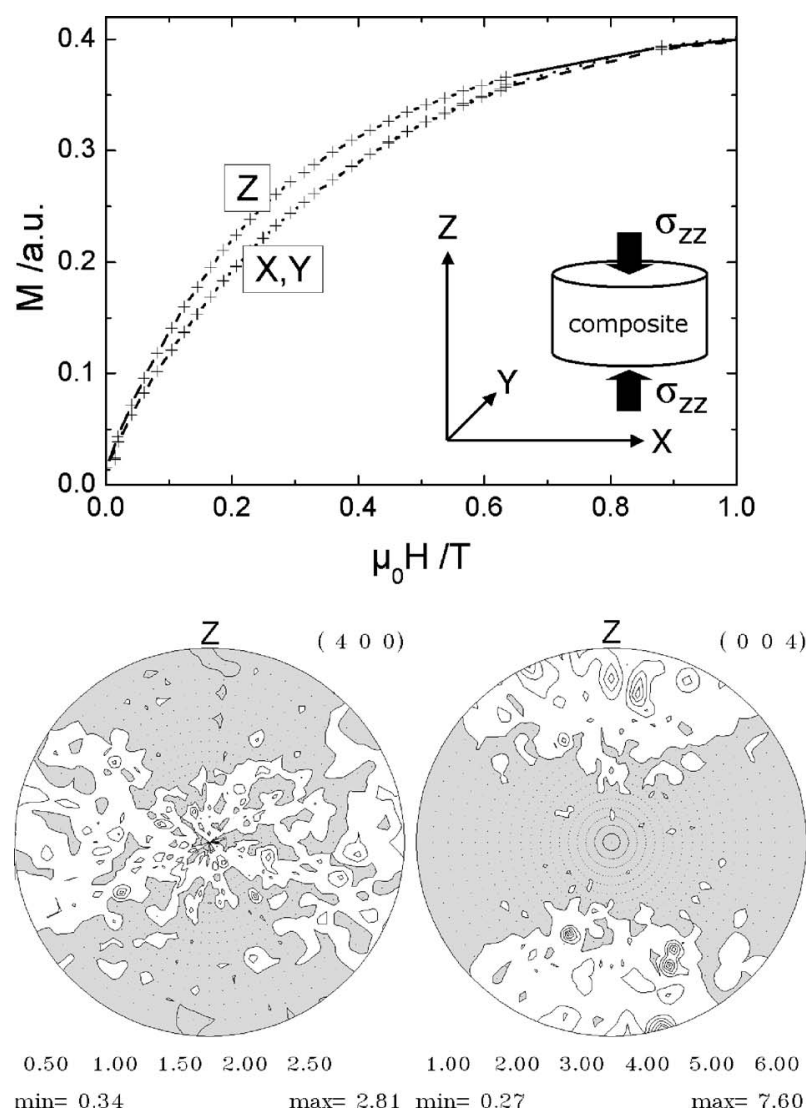

FIG. 2. Composite sample after compression along $Z$. The sample is now easier to magnetize in $Z$ direction than in the other two perpendicular directions (top). A (004)-fiber texture along the $Z$ direction is seen in the pole figures (bottom), verifying that the $c$ axis aligns with the compression direction $Z$.

number of particles rotate equally). Additionally, in situ deformation using an optical microscope did not show any particle rotation within the matrix. ${ }^{6}$ Thus, compression of the composite leads to a size reduction of the MSM particles in compression direction due to the stress induced twin boundary motion within the MSM particles.

MSM composites were prepared by embedding partly single-crystalline $\mathrm{Ni}_{50.9} \mathrm{Mn}_{27.1} \mathrm{Ga}_{22.0}$ particles in a polyester matrix. The $M-H$ curves and the texture of the MSM particles before and after compression were measured by vibrating sample magnetometry and synchrotron radiation, respectively. The composite's compressed direction becomes easier to magnetize and shows clearly a (004)-fiber texture. This confirms unambiguously stress induced twin boundary motion within the MSM particles. This energy dissipating process makes MSM composites suitable for magnetically controlled mechanical energy dampers.

This work is supported by PLANSEE SE (6600 Reutte, Austria) and DFG (SPP 1239). Experimental assistance by B. Gebel, K. Berger, and I. Huensche is gratefully acknowledged.

${ }^{1}$ K. Ullakko, J. K. Huang, C. Kantner, R. C. O'Handley, and V. V. Kokorin, Appl. Phys. Lett. 69, 1966 (1996).

${ }^{2}$ A. N. Vasil'ev, V. D. Buchel'nikov, T. Takagi, V. V. Khovailo, and E. I. Estrin, Phys. Usp. 46, 559 (2003).

${ }^{3}$ M. A. Marioni et al., J. Magn. Magn. Mater. 290-291, 35 (2005). 
${ }^{4}$ A. Sozinov, A. A. Likhachev, N. Lanska, and K. Ullakko, Appl. Phys. Lett. 80, 1746 (2002).

${ }^{5}$ J. Feuchtwanger, M. L. Richard, Y. J. Tang, A. E. Berkowitz, R. C. O'Handley, and S. M. Allen, J. Appl. Phys. 97, 10M319 (2005).

${ }^{6}$ N. Scheerbaum, D. Hinz, O. Gutfleisch, K.-H. Müller, and L. Schultz, Acta Mater. (in press).

${ }^{7}$ D. Hinz, N. Scheerbaum, O. Gutfleisch, K.-H. Müller, and L. Schultz, J. Magn. Magn. Mater. (in press).
${ }^{8}$ W. Skrotzki, B. Klöden, R. Tamm, C.-G. Oertel, U. Garbe, and E. Rybacki, Textures Microstruct. 35, 163 (2003).

${ }^{9}$ W. Skrotzki, B. Klöden, N. Scheerbaum, R. Tamm, C.-G. Oertel, U. Garbe, E. Rybacki, and H.-G. Brokmeier, "Local texture measurements with high-energy synchrotron radiation," in Proceedings of the International Seminar on Advanced X-Ray Techniques in Research and Industry, Hyderabad 2003, edited by A. K. Singh (Capital Publishing Company, New Delhi, 2006), pp. 33-49. 